\title{
Suction Failure
}

National Cancer Institute

\section{Source}

National Cancer Institute. Suction Failure. NCI Thesaurus. Code C133628.

Problem associated with the complete inability to provide suction. 